\title{
POSTER
}

\section{Tumeurs des glandes salivaires accessoires labiales : Étude d'une série de cas}

\section{Rochefort $\mathrm{J}^{1}$, Mohammad AK' ${ }^{1}$, Hervé $\mathrm{G}^{2}$, Agbo-godeau $\mathrm{S}^{3}$}

1 - Service d'Odontologie du Pr Descroix, Groupe Hospitalier Pitié Salpêtrière-Charles Foix, UFR Odontologie Université Paris Diderot

2 - Service d'Anatomie et de Cytologie pathologique du Pr Capron, Groupe Hospitalier Pitié Salpêtrière- Charles Foix, Université Pierre et Marie Curie

3 - Service de Stomatologie et Chirurgie Maxillo-Faciale du Pr Goudot, Groupe Hospitalier Pitié Salpêtrière-Charles Foix, Université Pierre et Marie Curie

\section{Introduction}

Les glandes salivaires accessoires (GSA) sont des glandes exocrines, de petite taille, très nombreuses, dispersées dans la muqueuse buccale au niveau des joues, des lèvres, de la langue et du palais [1]. Les tumeurs des GSA touchent plus souvent les adultes et représentent 5 à 10\% de l'ensemble des tumeurs des glandes salivaires [2]. II existe sur ce sujet peu d'études cliniques comportant des séries importantes [2], et les publications rapportent souvent des cas cliniques isolés. Ces tumeurs peuvent se présenter sous différentes formes anatomopathologiques, la classification OMS 2005 [1] distingue au sein des tumeurs primitives des glandes salivaires, 12 sous-types de tumeurs bénignes dont plusieurs types d'adénomes [3], et 24 sous-types de carcinomes [4]. Les présentations cliniques sont variées selon le type de tumeur [2].

\section{Matériel et méthode}

Notre étude rétrospective est basée sur le recueil de l'ensemble des données cliniques et histologiques des patients ayant été opérés d'un nodule tumoral salivaire labial au sein de l'hôpital Pitié Salpêtrière ces 10 dernières années. L'objectif de ce travail est d'établir la proportion de tumeurs malignes retrouvées dans les nodules labiaux de notre série et de la comparer aux données de la littérature.

\section{Discussion}

Bien que la nature maligne d'une tumeur se suspecte cliniquement par des signes objectivables comme l'adhérence aux plans profonds ou des limites floues, certaines tumeurs malignes peuvent, à leur stade débutant, paraître d'aspect bénin notamment celles qui atteignent les glandes salivaires accessoires des lèvres. C'est l'analyse histologique de la pièce opératoire obtenue par une exérèse simple qui en précise la nature. Les données de la littérature estiment, selon les études, une proportion de $28 \%$ à $50 \%$ d'atteinte maligne dans les tumeurs des glandes salivaires accessoires de la cavité orale [2]. 


\section{Conclusion}

Cette étude permettra d'évaluer la proportion de malignité des tumeurs des glandes salivaires labiales et en fonction de ces résultats de définir une stratégie de prise en charge diagnostique et thérapeutique de ces nodules labiaux.

\section{Références}

[1] Barnes L, Eveson JW, Reichart P, and Sidransky D, "Pathology and Genetics of Head and Neck Tumours In WHO Clasification of Tumours." IARC Press, 2005.

[2] P. Bonfils, "Tumeurs des glandes salivaires," EMC - Oto-Rhino-Laryngol., vol. 2, no. 2, pp. 1-18, Jan. 2007.

[3] P. Pitak-Arnnop, K. Dhanuthai, A. Hemprich, and N. C. Pausch, "Pleomorphic adenoma of the upper lip: some clinicopathological considerations," J. Cutan. Aesthetic Surg., vol. 5, no. 1, pp. 51-52, Jan. 2012.

[4] C. Badoual, J. Cros, H. Roussel, M. Wassef, and J. Cucherousset, "Les carcinomes des glandes salivaires : description histologique des principaux sous-types histologiques," RFL - Rev. Francoph. Lab., vol. 43, no. 448, pp. 55-61, Feb. 2013. 